\title{
Vertical muon intensity measured with MACRO at the Gran Sasso laboratory
}

\author{
M. Ambrosio, ${ }^{12}$ R. Antolini, ${ }^{7}$ G. Auriemma, ${ }^{14, *}$ R. Baker, ${ }^{11}$ A. Baldini, ${ }^{13}$ G. C. \\ Barbarino, ${ }^{12}$ B. C. Barish, ${ }^{4}$ G. Battistoni, ${ }^{6}, \dagger$ R. Bellotti, ${ }^{1}$ C. Bemporad,${ }^{13}$ P. \\ Bernardini, ${ }^{10}$ H. Bilokon, ${ }^{6}$ V. Bisi,${ }^{16}$ C. Bloise ${ }^{6}$ C. Bower, ${ }^{8}$ S. Bussino,${ }^{14} \mathrm{~F}$. \\ Cafagna, ${ }^{1}$ M. Calicchio, ${ }^{1}$ D. Campana, ${ }^{12}$ M. Carboni,${ }^{6}$ M. Castellano, ${ }^{1} \mathrm{~S}$. \\ Cecchini, ${ }^{2, \ddagger}$ F. Cei,${ }^{13, \S}$ P. Celio, ${ }^{14}$ V. Chiarella, ${ }^{6}$ A. Corona, ${ }^{14}$ S. Coutu, ${ }^{11}$ G. De \\ Cataldo ${ }^{1}$ H. Dekhissi, ${ }^{2}, \|$ C. De Marzo, ${ }^{1}$ I. De Mitri, ${ }^{9}$ M. De Vincenzi, ${ }^{14},{ }^{\top}$ A. Di \\ Credico, ${ }^{7,14}$ O. Erriquez,${ }^{1}$ C. Favuzzi, ${ }^{1}$ C. Forti, ${ }^{6}$ P. Fusco, ${ }^{1}$ G. Giacomelli, ${ }^{2}$ G. \\ Giannini, ${ }^{13, * *}$ N. Giglietto,${ }^{1}$ M. Grassi,${ }^{13}$ A. Grillo, ${ }^{7}$ F. Guarino, ${ }^{12}$ P. \\ Guarnaccia ${ }^{1}$ C. Gustavino, ${ }^{7}$ A. Habig ${ }^{8}$ K. Hanson, ${ }^{11}$ A. Hawthorne ${ }^{8}$ R. \\ Heinz,$^{8}$ J. T. Hong, ${ }^{3}$ E. Iarocci, ${ }^{6, t \dagger}$ E. Katsavounidis, ${ }^{4}$ E. Kearns, ${ }^{3}$ S. \\ Kyriazopoulou, ${ }^{4}$ E. Lamanna,${ }^{14}$ C. Lane,${ }^{5}$ D. S. Levin, ${ }^{11}$ P. Lipari, ${ }^{14}$ R. Liu,${ }^{4}$ N. \\ P. Longley, ${ }^{4}$ M. J. Longo, ${ }^{11}$ Y. Lu, ${ }^{15}$ G. Ludlam,${ }^{3}$ G. Mancarella, ${ }^{10}$ G. \\ Mandrioli, ${ }^{2}$ A. Margiotta-Neri, ${ }^{2}$ A. Marini, ${ }^{6}$ D. Martello, ${ }^{10}$ A. Marzari-Chiesa, ${ }^{16}$ \\ M. N. Mazziotta, ${ }^{1}$ D. G. Michael ${ }^{4}$ S. Mikheyev,${ }^{7, \ddagger \ddagger}$ L. Miller, ${ }^{8}$ M. Mittelbrunn, ${ }^{5}$ \\ P. Monacelli, ${ }^{9}$ T. Montaruli, ${ }^{1}$ M. Monteno, ${ }^{16}$ S. Mufson, ${ }^{8}$ J. Musser ${ }^{8}$ D. \\ Nicoló, ${ }^{13}, \S$ R. Nolty, ${ }^{4}$ C. Okada,${ }^{3}$ C. Orth,${ }^{3}$ G. Osteria, ${ }^{12}$ O. Palamara, ${ }^{10} \mathrm{~S}$. \\ Parlati, ${ }^{7}$ V. Patera, ${ }^{6, \dagger \dagger}$ L. Patrizii, ${ }^{2}$ R. Pazzi, ${ }^{13}$ C. W. Peck, ${ }^{4}$ S. Petrera, ${ }^{10}$ N. D. \\ Pignatano, ${ }^{4}$ P. Pistilli, ${ }^{10}$ V. Popa,${ }^{2, \S \S}$ A. Rainó,${ }^{1}$ J. Reynoldson, ${ }^{7}$ F. Ronga,${ }^{6}$ A. \\ Sanzgiri, ${ }^{15}$ F. Sartogo, ${ }^{14}$ C. Satriano, ${ }^{14, *}$ L. Satta,,${ }^{6 \dagger \dagger}$ E. Scapparone, ${ }^{2}$ K. \\ Scholberg, ${ }^{4}$ A. Sciubba, ${ }^{6, \dagger \dagger}$ P. Serra-Lugaresi, ${ }^{2}$ M. Severi,${ }^{14}$ M. Sitta, ${ }^{16}$ P. \\ Spinelli, ${ }^{1}$ M. Spinetti, ${ }^{6}$ M. Spurio, ${ }^{2}$ R. Steinberg, ${ }^{5}$ J. L. Stone ${ }^{3}$ L. R. Sulak ${ }^{3}$ A. \\ Surdo, ${ }^{10}$ G. Tarlé, ${ }^{11}$ F. Tassoni, ${ }^{14}$ V. Togo,${ }^{2}$ V. Valente,${ }^{6}$ C. W. Walter,${ }^{4}$ \\ and R. Webb ${ }^{15}$ \\ (MACRO Collaboration) \\ ${ }^{1}$ Dipartimento di Fisica dell'Università di Bari and Istituto Nazionale di Fisica Nucleare, 70126 Bari, Italy \\ ${ }^{2}$ Dipartimento di Fisica dell'Università di Bologna and Istituto Nazionale di Fisica Nucleare, 40126 Bologna, Italy \\ ${ }^{3}$ Physics Department, Boston University, Boston, Massachusetts 02215, \\ ${ }^{4}$ California Institute of Technology, Pasadena, California 91125, \\ ${ }^{5}$ Department of Physics, Drexel University, Philadelphia, Pennsylvania 19104 \\ ${ }^{6}$ Laboratori Nazionali di Frascati dell'Istituto Nazionale di Fisica Nucleare, 00044 Frascati (Roma), Italy \\ ${ }^{7}$ Laboratori Nazionali del Gran Sasso dell'Istituto Nazionale di Fisica Nucleare, 67010 Assergi (L'Aquila), Italy \\ ${ }^{8}$ Departments of Physics and of Astronomy, Indiana University, Bloomington, Indiana 47405 \\ ${ }^{9}$ Dipartimento di Fisica dell'Università dell'Aquila and Istituto Nazionale di Fisica Nucleare, 67100 L'Aquila, Italy \\ ${ }^{10}$ Dipartimento di Fisica dell'Università di Lecce and Istituto Nazionale di Fisica Nucleare, 73100 Lecce, Italy \\ ${ }^{11}$ Department of Physics, University of Michigan, Ann Arbor, Michigan 48109 \\ ${ }^{12}$ Dipartimento di Fisica dell'Università di Napoli and Istituto Nazionale di Fisica Nucleare, 80125 Napoli, Italy \\ ${ }^{13}$ Dipartimento di Fisica dell'Università di Pisa and Istituto Nazionale di Fisica Nucleare, 56010 Pisa, Italy \\ ${ }^{14}$ Dipartimento di Fisica dell'Università di Roma "La Sapienza" and Istituto Nazionale di Fisica Nucleare, o0185 Roma, Italy \\ ${ }^{15}$ Physics Department, Texas A 63 University, College Station, Texas 77843 \\ ${ }^{16}$ Dipartimento di Fisica Sperimentale dell'Università di Torino and Istituto Nazionale di Fisica Nucleare, 10125 Torino, Italy
} (Received 30 January 1995; revised manuscript received 3 April 1995)

The vertical underground muon intensity has been measured in the slant depth range 3200-7000 $\mathrm{hg} \mathrm{cm}^{-2}$ (standard rock) with the completed lower part of the MACRO detector at the Gran Sasso

\footnotetext{
*Also at Università della Basilicata, 85100 Potenza, Italy.

${ }^{\dagger}$ Also at INFN Milano, 20133 Milano, Italy.

$\ddagger$ Also at Istituto TESRE/CNR, 40129 Bologna, Italy.

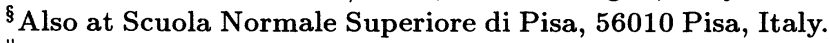

"Also at Faculty of Sciences, University Mohamed I, B.P. 424 Oujda, Morocco.

${ }^{\pi}$ Also at Dipartimento di Fisica, Università di Roma III, Roma, Italy.
}

\footnotetext{
**Also at Università di Trieste and INFN, 34100 Trieste, Italy.

${ }^{\dagger \dagger}$ Also at Dipartimento di Energetica, Università di Roma, 00185 Roma, Italy.

$¥$ Also at Institute for Nuclear Research, Russian Academy of Science, 117312 Moscow, Russia.

${ }_{\S}$ Also at Institute for Atomic Physics, 76900 Bucharest, Romania.
} 
laboratory, using a large sample of data. These observations are used to compute the surface muon flux and the primary "all-nucleon" spectrum. An analysis of systematic uncertainties introduced by the interaction models in the atmosphere and the underground propagation of muons is presented. A comparison of our results with published data is also presented.

PACS number(s): 13.85.Tp, 96.40.Tv

\section{INTRODUCTION}

In this work we present a new measurement of the inclusive flux of underground muons performed with the Monopole, Astrophysics, and Cosmic Ray Observatory (MACRO) detector running at the Gran Sasso National Laboratory in central Italy.

Muons detected in deep underground detectors can provide information on the spectrum and composition of primary cosmic rays [1] in the energy range $E_{0} \simeq 10^{14}$ $10^{16} \mathrm{eV}$. The most sensitive measurements are obtained by considering the frequency of events as a function of the muon multiplicity [2]. For this purpose it is necessary to have detectors with geometrical dimensions large with respect to the average separation [3] of underground muons. The sensitivity of the measurements is improved if the measurement of the muon multiplicity underground is accompanied by the coincident measurement of other properties of the primary particle induced shower [4]. In the measurement of the inclusive muon intensity that is discussed here, one considers the total flux of muons observed underground for different slanted depths and different zenith angles, summing over all muon multiplicities, without considering correlations with other measurements of the primary particle shower. In this way some amount of information is lost; however, this measurement is interesting for several reasons: The complications of determining the detector acceptance for multiple muon events [3] are absent, the inclusive muon flux has been measured before with smaller detectors, and our results can be compared to these earlier measurements. The inclusive muon flux can be related to the inclusive flux of primary nucleons, i.e., the flux of nucleons obtained summing over all primary masses. In fact the composition of the primary cosmic rays is important in determining the multiplicity distribution of the underground muon events, but has a negligible effect on the inclusive muon flux.

The determination of the "all-nucleon" flux obtained with this technique is competitive with measurements obtained with direct [5] and indirect [6] methods that are limited, respectively, by statistical and systematic uncertainties. On the other hand a comparison of the "all-nucleon" primary flux determined from the underground muon intensity with the results obtained with other methods is a sensitive test of the models of muon production in hadronic showers that are used to study the cosmic ray composition [2].

We shall discuss measurements of the underground muon flux in the range of depth $3200 \leq h \leq 7000$ $\mathrm{hg} \mathrm{cm}{ }^{-2}$. The minimum depth is determined by the location of the underground laboratory where the MACRO detector is located. The region of very large depths, including the region where neutrino interactions in the vicinity of the detector become the dominant source of muons, will be discussed in a future paper.

The measured muon flux in the slant depth region considered corresponds to the muon spectrum at the surface in the energy range $1-20 \mathrm{TeV}$. The corresponding allnucleon primary flux is in the energy range $2-200 \mathrm{TeV}$.

Results on the underground muon intensity obtained by the MACRO Collaboration with a limited portion of the detector in operation were presented in [7]. In the present work we discuss a data sample 10 times larger and give a more complete discussion of the systematic effects that dominate the uncertainty in the measurement.

The paper is organized as follows: in Sec. II we briefly discuss the detector and the data selection criteria used; in Sec. III we present our determination of the underground muon intensity; in Sec. IV we use the results to estimate the all-nucleon primary spectrum considering uncertainties in the modeling of muon production; in Sec. $\mathrm{V}$ we discuss the sea-level muon spectrum $\left(E_{\mu} \geq 1\right.$ $\mathrm{TeV}$ ) implied by our measurement and compare the results with earlier measurements $[8,9]$.

\section{DETECTOR AND DATA SELECTION}

The present analysis was carried out on data collected with the completed lower part of the MACRO detector, with an acceptance of $S \Omega \simeq 3100 \mathrm{~m}^{2} \mathrm{sr}$ for atmospheric muons. The lower structure consists of six nearly identical units, called supermodules, of $12 \mathrm{~m} \times 12 \mathrm{~m} \times 4.8$ $\mathrm{m}$. Each supermodule, described in detail elsewhere [10], consists of ten horizontal planes of streamer tubes. The eight innermost planes are separated by seven layers, each of $\simeq 60 \mathrm{~g} \mathrm{~cm}^{-2}$ absorbers of low activity Gran Sasso rock. The two outermost planes are separated by two $19 \mathrm{~cm}$ layers of liquid scintillators. The lateral walls consist of stacked tanks of liquid scintillator, $25 \mathrm{~cm}$ thick, sandwiched between six vertical streamer tubes planes.

All streamer tube wires are read out, providing the $X$ coordinate on the horizontal planes and the $Z$ coordinate on the vertical planes. On the horizontal planes the second coordinate $D$ is obtained by reading the pulses induced on horizontal aluminum strips oriented at $26.5^{\circ}$ with respect to the streamer tubes axis, to allow stereoscopic reconstruction. Muon tracks are thus reconstructed with an angular resolution of $0.2^{\circ}$. The systematic uncertainty in the zenith angle reconstruction has been carefully checked for muon astronomy measurements and is less than $0.25^{\circ}$ [11]. This resolution is negligible compared to the average multiple scattering angle of $0.8^{\circ}$ for muons crossing the overburden rock. This value is consistent with the angular differences measured between muons belonging to the same event detected in MACRO. 
Data were collected starting in July 1991, after the completion of the lower part of MACRO while the upper part was still under construction; use was made of only the streamer tube system of the completed lower part. The hardware trigger was defined by either six streamer tube planes fired anywhere, or five consecutive horizontal planes, excluding the first and the last ones. A muon track is reconstructed if at least four horizontal planes are recorded, both in the wire and strip views. The data runs were then selected as follows: Runs were accepted if they had $\geq 4 \mathrm{~h}$ duration, and had a dead time smaller than $1 \%$ and a counting rate per hour per supermodule inside a range of $\pm 3 \sigma$ around the mean value simultaneously for all the six supermodules. These requirements assure a full and uniform acceptance of the apparatus. After these cuts, we have $3.91 \times 10^{6}$ muons for a live time of $4228 \mathrm{~h}$. These statistics are more than one order of magnitude larger than those reported in [7]. The large statistics allows us to study the Gran Sasso rock systematics and to reject angular regions where the muon intensities are not compatible with the measured average intensities of the regions with the same nominal rock thickness, as described in the Appendix.

\section{VERTICAL MUON INTENSITY}

The total data sample (single and multiple muons) is used to determine the bin-by-bin muon intensity $I_{\mu}(h, \theta, \phi)$ as

$$
I_{\mu}(h, \theta, \phi)=\left(\frac{1}{\Delta T}\right) \frac{\Sigma_{i} N_{i} m_{i}}{\Sigma_{j} \Delta \Omega_{j} A_{j} \epsilon_{j}},
$$

where $\Delta T$ is the live time, $N_{i}$ is the number of observed events of muon multiplicity $m_{i}$ in the angular bin $\Delta \Omega_{j}$ of slant depth $h$ (taken from the military topographical map of the mountain, as described in the Appendix), $A_{j}$ is the geometric detector projected area for that bin, $\epsilon_{j}$ is the combined trigger and reconstruction efficiency, $\theta$ is the muon zenith angle, and $\phi$ is the azimuth angle. The data are binned with $\Delta \theta=1^{\circ}, \Delta \phi=2^{\circ}$.

The projected area $A_{j}(\theta, \phi)$ and the detector tracking efficiency $\epsilon_{j}(\theta, \phi)$ were calculated with accuracies better than $1 \%$ from a Monte Carlo program, based on GEANT [12] to produce simulated data which were processed through the same off-line program chain used for the real data. All sources of inefficiencies (detector, electronics, and trigger) are included in the Monte Carlo program. The product $A_{j} \times \epsilon_{j}$ is shown in Fig. 1 as a function of the polar and azimuthal angles.

For each bin the Gran Sasso rock thickness (in meters) was converted to standard rock slant depth (in $\mathrm{hg} \mathrm{cm}^{-2}$ ) using the Gran Sasso rock parameters listed in Table IV, below, and a conversion formula described in Ref. [13].

In order to compare our results with those of other experiments, we calculated the vertical muon intensity using the well-known $\sec (\theta)$ angular dependence (valid up to $\left.60^{\circ}[1]\right)$ :

$$
I_{\mu}^{v}(h, \theta, \phi)=\left(\frac{1}{\Delta T}\right) \frac{\Sigma_{i} N_{i} m_{i}}{\Sigma_{j} \Delta \Omega_{j} A_{j} \epsilon_{j} / \cos \theta_{j}} .
$$

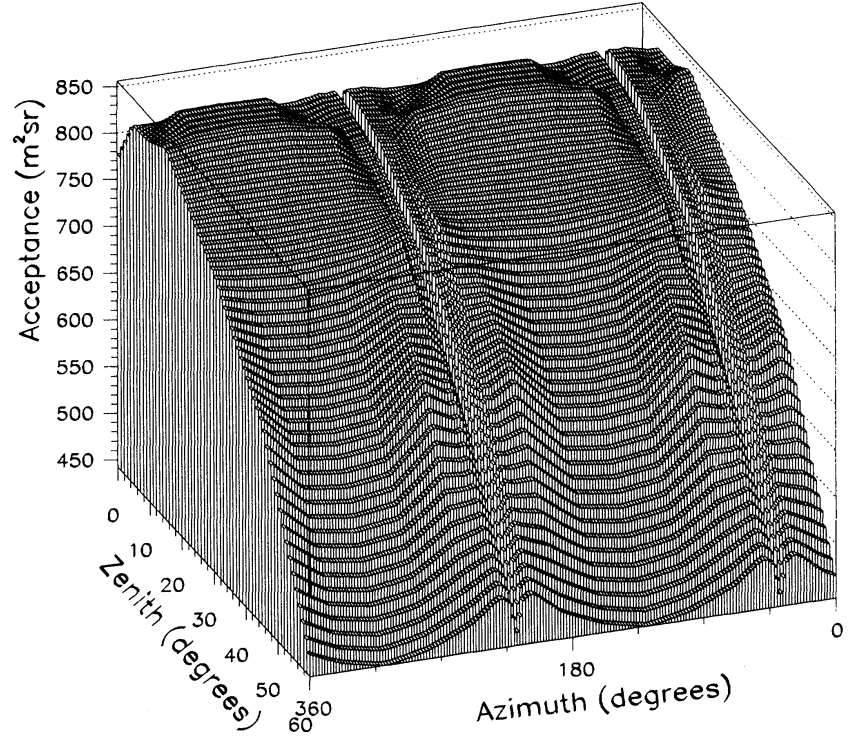

FIG. 1. Projected area $A_{j}$ times efficiency $\epsilon_{j}$ of the detector versus geographical coordinates. The polar angle extends up to $60^{\circ}$.

We have considered 54 bins of equal slant depth $h$, of width $\Delta h=50 \mathrm{hg} \mathrm{cm}^{-2}$ for the range $3200<h<$ $4750 \mathrm{hg} \mathrm{cm}^{-2}$; for the range $4750<h<6950 \mathrm{hg} \mathrm{cm}^{-2}$ we have used bins of $\Delta h=100 \mathrm{hg} \mathrm{cm}^{-2}$. The measured underground vertical muon intensity as a function of the slant depth $h$, for the zenith range $0^{\circ}-60^{\circ}$, is given in Table $I$ and is shown in Fig. 2. Each point is the mean value of the $I_{\mu}^{v}(h, \theta, \phi)$ distribution at fixed slant depth $h$.

We explored the effects of the main sources of systematics. The use of an average rock density (estimated using the results of the bore hole surveys of the mountain) instead of a function depending on the zenithal and azimuthal angles contributes an uncertainty of about $\pm 1.5 \%$ to the rock thickness, corresponding to $\pm 5 \%$ on the muon intensity at $3200 \mathrm{hg} \mathrm{cm}^{-2}$. A further $5 \%$ contribution to the absolute scale of the muon intensity comes from the assumption of a homogeneous mountain instead of a layered structure as modeled in Ref. [14] and described in [15]. The total systematic uncertainty is estimated at $\pm 8 \%$.

In the range $3200-7000 \mathrm{hg} \mathrm{cm}^{-2}$ our data are well fitted by the three-parameter empirical formula

$$
I_{\mu}(h)=A\left(\frac{h_{0}}{h}\right)^{\alpha} e^{-\frac{h}{h_{0}}},
$$

with $A=(1.96 \pm 0.03) \times 10^{-6} \mathrm{~cm}^{-2} \mathrm{~s}^{-1} \mathrm{sr}^{-1}, \alpha=1.10$ \pm 0.01 , and $h_{0}=(972 \pm 3) \mathrm{hg} \mathrm{cm}^{-2}$ with a $\chi^{2} / N_{\mathrm{DF}}=$ $65 / 51$. Using the Frejus function [16]

$$
I_{\mu}(h)=B\left(\frac{h_{1}}{h}\right)^{2} e^{-\frac{h}{h_{1}}},
$$

we obtain $B=(1.81 \pm 0.06) \times 10^{-6} \mathrm{~cm}^{-2} \mathrm{~s}^{-1} \mathrm{sr}^{-1}$ and $h_{1}=(1231 \pm 1) \mathrm{hg} \mathrm{cm}^{-2}$ with a $\chi^{2} / N_{\mathrm{DF}}=76 / 52$. The errors quoted for the fitted parameters include statisti- 
TABLE I. Measured vertical muon underground intensity $I_{\mu}(h)$ (muons $\mathrm{cm}^{-2} \mathrm{~s}^{-1} \mathrm{sr}^{-1}$ ) versus slant depth of standard rock $\left(\mathrm{hg} \mathrm{cm}^{-2}\right)$. The quoted errors include statistical uncertainties and systematic uncertainties for the topographical map. The additional estimated systematic scale uncertainty is $\pm 8 \%$; see text.

\begin{tabular}{lccc}
\hline \hline Depth & $I(h) \pm \Delta I(h)$ & Depth & $I(h) \pm \Delta I(h)$ \\
\hline 3200 & $(2.00 \pm 0.01) \times 10^{-8}$ & 4550 & $(3.29 \pm 0.07) \times 10^{-9}$ \\
3250 & $(1.85 \pm 0.01) \times 10^{-8}$ & 4600 & $(3.05 \pm 0.07) \times 10^{-9}$ \\
3300 & $(1.73 \pm 0.01) \times 10^{-8}$ & 4650 & $(2.92 \pm 0.04) \times 10^{-9}$ \\
3350 & $(1.59 \pm 0.01) \times 10^{-8}$ & 4700 & $(2.72 \pm 0.06) \times 10^{-9}$ \\
3400 & $(1.48 \pm 0.01) \times 10^{-8}$ & 4762 & $(2.61 \pm 0.04) \times 10^{-9}$ \\
3450 & $(1.39 \pm 0.01) \times 10^{-8}$ & 4850 & $(2.32 \pm 0.06) \times 10^{-9}$ \\
3500 & $(1.30 \pm 0.01) \times 10^{-8}$ & 4950 & $(2.02 \pm 0.06) \times 10^{-9}$ \\
3550 & $(1.215 \pm 0.008) \times 10^{-8}$ & 5050 & $(1.86 \pm 0.03) \times 10^{-9}$ \\
3600 & $(1.144 \pm 0.008) \times 10^{-8}$ & 5150 & $(1.60 \pm 0.04) \times 10^{-9}$ \\
3650 & $(1.058 \pm 0.007) \times 10^{-8}$ & 5250 & $(1.40 \pm 0.02) \times 10^{-9}$ \\
3700 & $(1.000 \pm 0.007) \times 10^{-8}$ & 5350 & $(1.28 \pm 0.04) \times 10^{-9}$ \\
3750 & $(9.44 \pm 0.07) \times 10^{-9}$ & 5450 & $(9.6 \pm 0.2) \times 10^{-10}$ \\
3800 & $(8.85 \pm 0.06) \times 10^{-9}$ & 5550 & $(8.7 \pm 0.2) \times 10^{-10}$ \\
3850 & $(8.23 \pm 0.06) \times 10^{-9}$ & 5650 & $(7.5 \pm 0.1) \times 10^{-10}$ \\
3900 & $(7.73 \pm 0.07) \times 10^{-9}$ & 5750 & $(6.8 \pm 0.2) \times 10^{-10}$ \\
3950 & $(7.20 \pm 0.07) \times 10^{-9}$ & 5850 & $(5.8 \pm 0.5) \times 10^{-10}$ \\
4000 & $(6.75 \pm 0.06) \times 10^{-9}$ & 5950 & $(5.2 \pm 0.3) \times 10^{-10}$ \\
4050 & $(6.37 \pm 0.06) \times 10^{-9}$ & 6050 & $(4.6 \pm 0.2) \times 10^{-10}$ \\
4100 & $(5.88 \pm 0.06) \times 10^{-9}$ & 6150 & $(4.3 \pm 0.1) \times 10^{-10}$ \\
4150 & $(5.49 \pm 0.06) \times 10^{-9}$ & 6250 & $(3.6 \pm 0.7) \times 10^{-10}$ \\
4200 & $(5.15 \pm 0.05) \times 10^{-9}$ & 6350 & $(3.2 \pm 0.4) \times 10^{-10}$ \\
4250 & $(4.82 \pm 0.06) \times 10^{-9}$ & 6450 & $(2.7 \pm 0.3) \times 10^{-10}$ \\
4300 & $(4.51 \pm 0.05) \times 10^{-9}$ & 6550 & $(2.7 \pm 0.5) \times 10^{-10}$ \\
4350 & $(4.21 \pm 0.07) \times 10^{-9}$ & 6650 & $(2.2 \pm 0.3) \times 10^{-10}$ \\
4400 & $(3.94 \pm 0.07) \times 10^{-9}$ & 6750 & $(2.2 \pm 0.3) \times 10^{-10}$ \\
4450 & $(3.69 \pm 0.09) \times 10^{-9}$ & 6850 & $(2.0 \pm 0.3) \times 10^{-10}$ \\
4500 & $(3.46 \pm 0.03) \times 10^{-9}$ & 6950 & \\
\hline \hline
\end{tabular}

cal uncertainties and point-to-point uncertainties in the Gran Sasso map.

Figure 3 shows the behavior of the intensity as a function of $\sec (\theta)$ at fixed depths. In Fig. 3(a) the data were binned in four depth regions of rock and in Fig. 3(b) the experimental points are scaled to the central slant

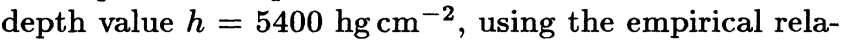
tion described in item (b) of Ref. [6]. The quoted errors include statistical and point-to-point uncertainties; the global systematic uncertainties related to the mountain knowledge are not included. The linear behavior of the data is an a posteriori confirmation of the angular dependence of the underground muon intensity.

In Fig. 4(a) our data are compared with the world data in the range $1000-17000 \mathrm{hg} \mathrm{cm}^{-2}$; Fig. $4(\mathrm{~b})$ is a blowup of the slant depth region relevant to our present results. Our data agree, within their combined statistical and systematic uncertainties, with the data of other experiments. In Fig. 4(b) our fit is compared to previous fits of other experiments and to the Crouch compilation presented in Ref. [17]. A difference of about $40 \%$ is found between MACRO and the Nucleon Stability Experiment (NUSEX), where the data overlap [8]. The Frejus fit [16] differs from ours by about 10-15\%, which corresponds to about one standard deviation of the Frejus fitted parameters. The comparison of our data with the Crouch compilation shows differences of less than $6 \%$. The ob- served discrepancies with NUSEX might be connected to unknown systematic uncertainties in their rock overburden.

\section{PRIMARY SPECTRUM}

In the context of the superposition model, the muon flux at the surface contains information on the "all-

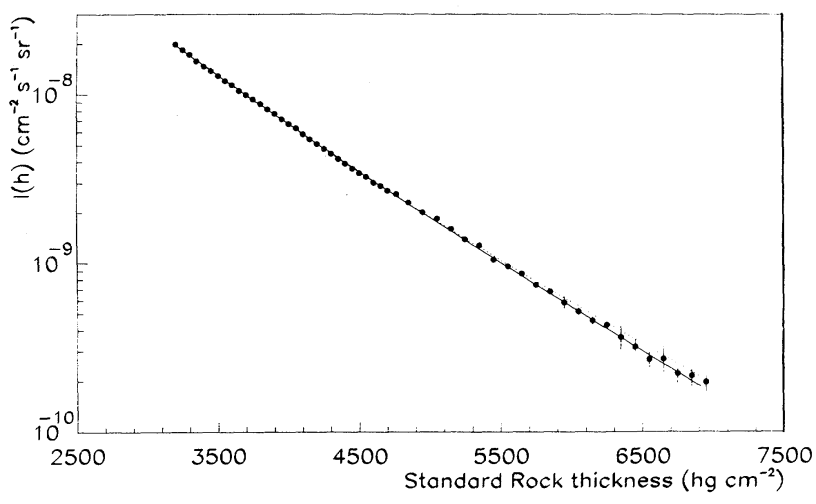

FIG. 2. Measured vertical muon intensity versus standard rock (black points). The dotted and solid lines are the twoand three-parameter fits described in the text. 
TABLE II. Spectrum weighted moments $Z_{i j}$ and atmospheric attenuation lengths $\Lambda_{i}\left(\right.$ in $\mathrm{g} \mathrm{cm}^{-2}$ ) for hadrons and primary all-nucleon spectrum coefficients obtained from the three fits described in the text.

\begin{tabular}{l|ccc|cc}
\hline \hline & \multicolumn{3}{|c}{ Input } & & \multicolumn{2}{c}{ Output } \\
Model & $Z_{N N}$ & $Z_{N \pi}$ & $Z_{N K}$ & $N_{0}\left(\mathrm{~cm}^{-2} \mathrm{~s}^{-1} \mathrm{sr}^{-1} \mathrm{GeV}^{\gamma_{p}-1} A\right)$ & $\gamma_{p}$ \\
\hline Gaisser & 0.298 & 0.079 & 0.0118 & $3.4 \pm 0.1$ & $2.78 \pm 0.04$ \\
HEMAS & 0.26 & 0.057 & 0.0113 & $5.0 \pm 0.1$ & $2.79 \pm 0.04$ \\
SIBYLL & 0.28 & 0.068 & 0.0071 & $4.1 \pm 0.1$ & $2.77 \pm 0.05$ \\
\hline \hline & $\Lambda_{N}$ & $\Lambda_{\pi}$ & $\Lambda_{K}$ & & \\
All models & 120 & 160 & 180 & & \\
\hline \hline
\end{tabular}

nucleon" primary spectrum $N\left(E_{p}\right)$. In the energy range relevant for the present measurement $\left(2<E_{p}<200\right.$ $\mathrm{TeV} /$ nucleon), the relation between the surface muon flux $\frac{d N_{\mu}}{d E d \Omega}$ and the "all-nucleon" primary spectrum $N\left(E_{p}\right)$ is approximated using the same relations used in Ref. [1] by the formula

$\frac{d N_{\mu}}{d E d \Omega}$

$$
\simeq N\left(E_{p}\right) \frac{Z_{N \pi}}{1-Z_{N N}} \frac{\left[1-\left(r_{\pi}\right)^{\gamma+1}\right]\left(1-r_{\pi}\right)^{-1}(\gamma+1)^{-1}}{1+\frac{B_{\pi} \cos \theta E}{\epsilon_{\pi}}}
$$

where

$$
\begin{aligned}
B_{\pi} & =\frac{(\gamma+2)}{(\gamma+1)} \frac{1-\left(r_{\pi}\right)^{\gamma+1}}{1-\left(r_{\pi}\right)^{\gamma+2}} \frac{\Lambda_{\pi}-\Lambda_{N}}{\Lambda_{\pi} \ln \left(\Lambda_{\pi} / \Lambda_{N}\right)} \\
r_{\pi} & =\left(\frac{m_{\mu}}{m_{\pi}}\right)^{2} .
\end{aligned}
$$
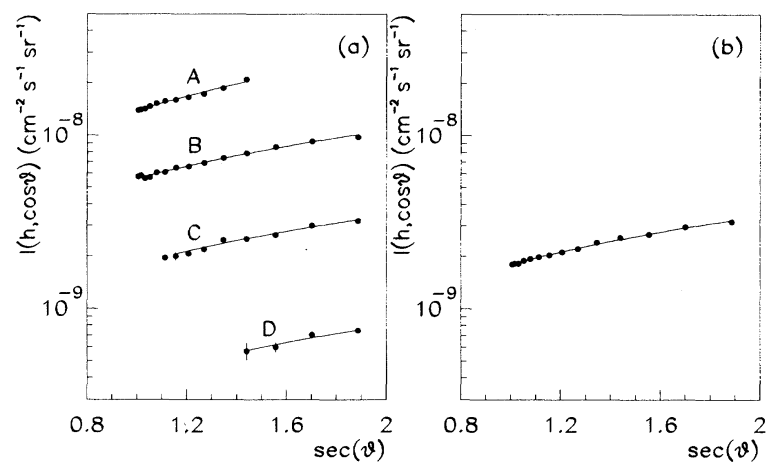

FIG. 3. (a) Muon intensity versus $1 / \cos (\theta)$ for four ranges of rock depth: $3150-3750(A), 3850-4550(B), 4650-5550(C)$, and $5650-6950 \mathrm{hg} \mathrm{cm}^{-2}$. (D) The data shown in (a) scaled to $h=5400 \mathrm{hg} \mathrm{cm} \mathrm{cm}^{-2}$. The solid lines are linear fits to our data. In the explored angular range $\left(0-60^{\circ}\right)$ and for the energies relevant to our experiment $\left(E_{\mu}>1 \mathrm{TeV}\right)$ we do not observe, within the experimental uncertainties, deviations from the conventional $\sec (\theta)$ approximation.
Equation (5) is summed over pion and kaon decay channels; $B_{K}$ and $r_{K}$ are defined in a similar fashion. The constant $\epsilon_{\pi, K}$ contains the meson lifetimes and depends on the structure of the atmosphere; $Z_{i j}$ are the spectrum averaged moments, which may depend on energy; they contain information on the inclusive distribution as well as on the primary spectrum; $\Lambda_{i}$ are the atmospheric attenuation lengths.

Assuming for the "all-nucleon" primary spectrum a simple power dependence
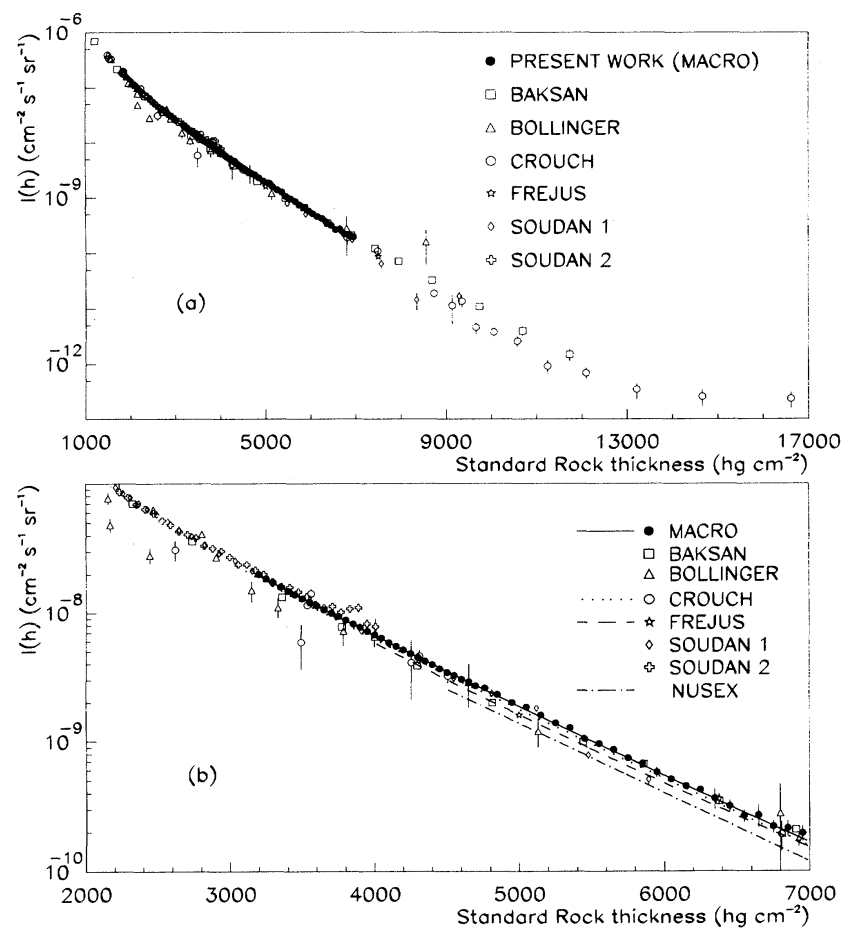

FIG. 4. Vertical muon intensity versus standard rock. (a) The present MACRO results, the data compiled by Crouch [17], and those obtained by other experiments: BAKSAN $[6(\mathrm{~g})]$, Bollinger [6(e)], Frejus [16], and Soudan 1 and Soudan 2 [18]. (b) The depth region covered by our data shown in more detail. The solid line is the fit of our data to Eq. (3), the dotted line is the Crouch fit [17], the dashed line is the Frejus fit, and the dash-dotted line the NUSEX fit. 


$$
N\left(E_{p}\right)=N_{0} E^{-\gamma_{p}}
$$

(well established in the energy range relevant to this measurement), both the spectral index $\gamma_{p}$ and the normalization $N_{0}$ can be derived from the vertical muon intensity, after the spectrum averaged moments and interaction lengths (as well as details of the atmosphere) are deduced from a Monte Carlo simulation. In the approximation of exact Feynman scaling and a single power spectrum, the spectrum averaged moments and interaction lengths are constant.

We estimated the "all-nucleon" primary spectrum by the least squares method unfolding $N\left(E_{p}\right)$ from the measured underground muon intensity $I_{\mu}(h)$ :

$$
I_{\mu}(h)=\int_{0}^{\infty} \frac{d N_{\mu}}{d E d \Omega} P(E, h) d E
$$

where $h$ is the rock depth, $\frac{d N_{\mu}}{d E d \Omega}$ is the muon intensity at the surface, and $P(E, h)$ is the survival probability; the integration is performed at constant slant depth values. The survival probabilities were calculated for surface muons with energies in the 1-100 $\mathrm{TeV}$ energy range, using a GEANT code especially tuned for the Gran Sasso rock. The code includes a detailed description of muon propagation underground and accounts for fluctuations in muon energy losses [15].

We used different sets of $Z_{i j}$ functions derived from three interaction models: (a) from Ref. [1], where the $Z_{i j}$ are constant as a function of energy since Feynman scaling is assumed to be exact, (b) the HEMAS interaction model [19], and (c) the SIBYLL interaction model [20]. In the latter two cases the $Z_{i j}$ functions exhibit a smooth dependence on primary energy, since scaling violations

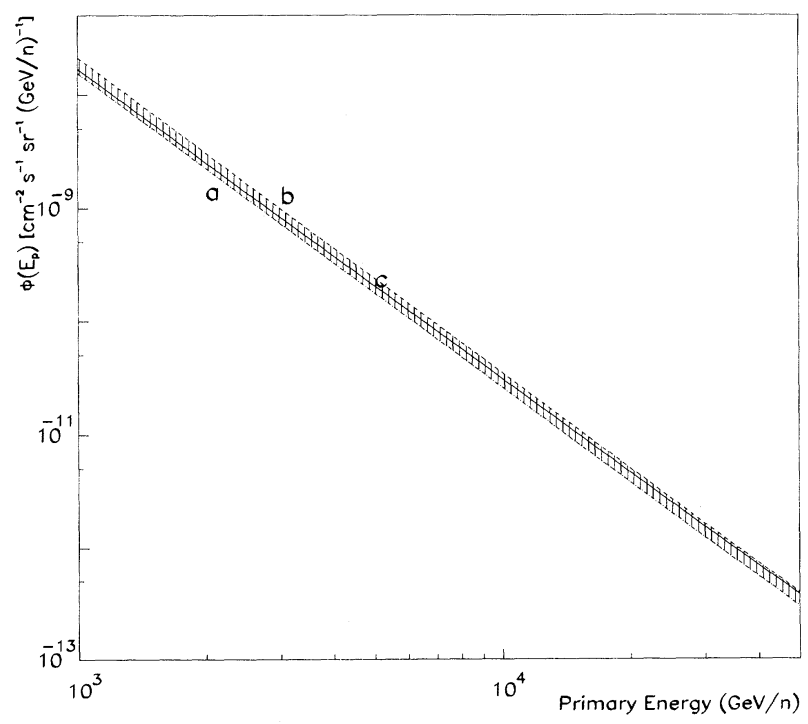

FIG. 5. "All-nucleon" primary spectrum $\Phi\left(E_{p}\right)$ versus energy $E_{p}$. Curves $a$ and $b$ : MACRO values according to the Gaisser (dash-dotted line) and to the HEMAs (dashed line) models (see Table II). Curve $c$ : Average of the direct measurements (solid line) $[5,22]$. The dashed area represents the region between the HEMAS and Gaisser models; see Sec. IV.
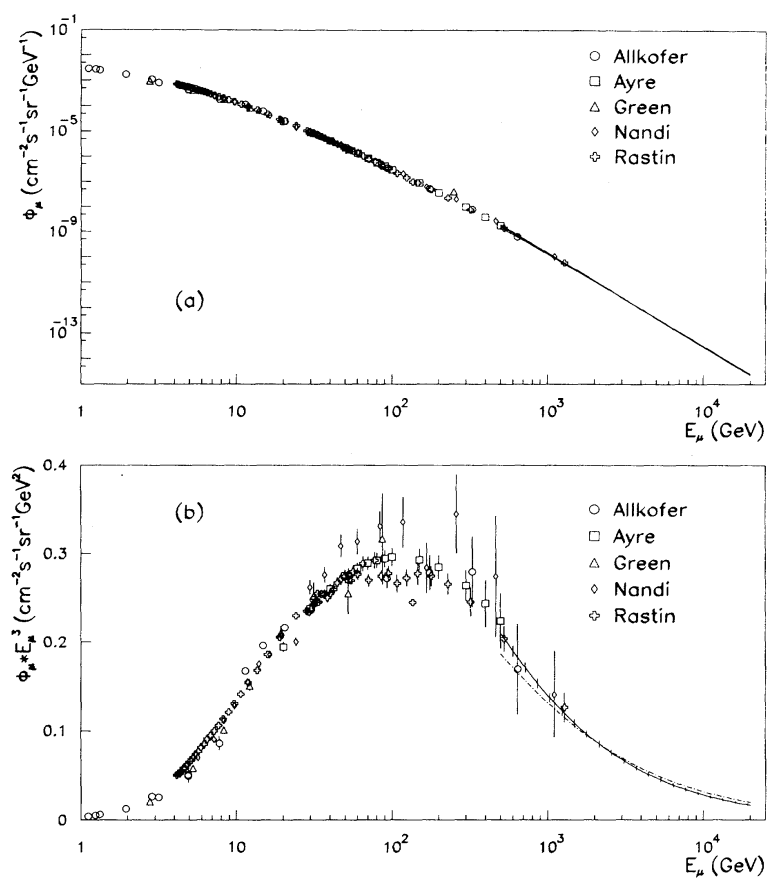

FIG. 6. (a) Differential muon energy flux at the surface, $\Phi_{\mu}$. The solid line is the MACRO fit; it is compared with the available experimental measurements: Allkofer et al. [28], Ayre et al. [29], Green et al. [30], Nandi and Sinha [31], and Rastin [32]. (b) $E_{\mu}^{3} \Phi_{\mu}\left(E_{\mu}\right)$ is shown as a function of $E_{\mu}$. The solid line shows the fit to formula (9); the dash-dotted line is from the formula on p. 71 of [1].

are included in the two models. As a reasonable approximation we have chosen the values at $10 \mathrm{TeV} /$ nucleon, since this is the most probable energy of primaries that produce the inclusive muon flux at MACRO depth. We have also made use of constant values for the attenuation lengths given in [1]. The numerical values of these parameters are listed in Table II.

The fit of our data using the three models gives the spectral index $\gamma_{p}$ and the normalization factor $N_{0}$ quoted in Table II. The correlation coefficient between $\gamma_{p}$ and $N_{0}$ is 0.975. The errors include both statistical and map resolution uncertainties. Further uncertainties of the order of $5 \%$ in $N_{0}$ and $3 \%$ in $\gamma_{p}$ should be considered as discussed in the next section. The spread of the three values gives an estimate of the uncertainties on the primary flux due to the interaction model. Our evaluations are in agreement with the estimates reported in [21]. The values

\begin{tabular}{|c|c|c|}
\hline Rock type & Chemical composition & $\begin{array}{c}\% \\
\text { weight }\end{array}$ \\
\hline Dolomite & $\mathrm{CaCO}_{3}(90 \%), \mathrm{MgCO}_{3}(10 \%)$ & 50 \\
\hline Dolomite limestone & $\mathrm{CaCO}_{3}(50 \%), \mathrm{MgCO}_{3}(50 \%)$ & 29 \\
\hline Flint limestone & $\begin{array}{c}\mathrm{CaCO}_{3}(72 \%), \mathrm{SiO}_{2}(8 \%) \\
\mathrm{Si}, \mathrm{Al}, \mathrm{K} \text { compounds }(20 \%)\end{array}$ & 8 \\
\hline Karst formation & $\mathrm{CaCO}_{3}$ & 9 \\
\hline Detritus & $\begin{array}{l}\mathrm{CaCO}_{3}(49 \%), \mathrm{MgCO}_{3}(1 \%) \\
\mathrm{Si}, \mathrm{Al}, \mathrm{K} \text { compounds }(50 \%)\end{array}$ & 3 \\
\hline
\end{tabular}

TABLE III. Gran Sasso rock chemical composition [14]. 
TABLE IV. Gran Sasso rock average parameters. They are very similar to the so-called standard rock for which $A=22, Z=11$, and $\rho=2.65 \mathrm{~g} \mathrm{~cm}^{-3}$.

\begin{tabular}{|c|c|c|c|}
\hline$A=22.87$ & $Z=11.41$ & Density $=(2.71 \pm 0.05) \mathrm{g} \mathrm{cm}^{-3}$ & \\
\hline Chemical element & Atomic number & Atomic weight & Relative weight \\
\hline Hydrogen & 1 & 1.008 & 0.03 \\
\hline Carbon & 6 & 12.011 & 12.17 \\
\hline Oxygen & 8 & 15.99 & 50.77 \\
\hline Magnesium & 12 & 24.305 & 8.32 \\
\hline Aluminum & 13 & 26.981 & 0.63 \\
\hline Silicon & 14 & 28.085 & 1.05 \\
\hline Potassium & 19 & 39.098 & 0.10 \\
\hline Calcium & 20 & 40.078 & 26.89 \\
\hline
\end{tabular}

corresponding to the Gaisser and the HEMAS models are compared in Fig. 5 with the average of the direct measurements in the energy range 1-50 TeV/nucleon given in $[5,22]$. The direct measurements are contained in the dashed region in Fig. 5 which represents the region of our estimate due to the uncertainties of the interaction models. A comment is in order. We notice that the $Z$ functions from HEMAS and SIBYLL, used to reproduce the observed underground muon intensity, produce a reconstructed all-nucleon spectrum higher than that obtained from the average of the existing direct measurements in the range $1-50 \mathrm{TeV} /$ nucleon $[5,22]$. This is consistent with the analysis of the muon multiplicity distributions by MACRO [23], where a full simulation using the HEMAS code gave an absolute rate of events $25 \%$ lower with respect to the experimental data.

\section{MUON FLUX AT THE SURFACE}

In order to evaluate the surface muon flux, we follow the same procedure used in Sec. IV, with the parameters of the model described in [1]:

$\frac{d N_{\mu}}{d E d \Omega}=A_{0} E^{-\gamma_{\mu}}\left(\frac{1}{1+\frac{1.1 E \cos \theta}{115 \mathrm{GeV}}}+\frac{0.054}{1+\frac{1.1 E \cos \theta}{850 \mathrm{GeV}}}\right)$

We obtain $A_{0}=(0.26 \pm 0.01) \mathrm{cm}^{-2} \mathrm{~s}^{-1} \mathrm{sr}^{-1} \mathrm{GeV}^{\gamma_{\mu}-1}$, $\gamma_{\mu}=2.78 \pm 0.01$ with a $\chi^{2} / N_{\mathrm{DF}}=41 / 52$. The quoted errors are due to statistics and the map resolution. The fitted parameters are also affected by systematic uncertainties coming from the rock density and the hard energy loss cross sections used to estimate the survival probabilities. The effect of the uncertainty in the rock density produces an estimated uncertainty of $3.5 \%$ in $A_{0}$ and less than $1 \%$ in $\gamma_{\mu}$. Because of uncertainties in the bremsstrahlung and photonuclear cross sections [24], the results depend upon the cross sections of the stochastic radiative processes used in GEANT. We used different sets of survival probabilities to test the sensitivity of the fitted parameters to these uncertainties. Using the energy losses of Ref. [25], where a different photoproduction cross section is employed [26], we obtain a variation of $\simeq 2 \%$ in both $A_{0}$ and $\gamma_{\mu}$ and a $\chi^{2} / N_{\mathrm{DF}}=2.9$. We estimate the overall systematic error resulting from rock density and hard energy loss cross sections to be about
$5 \%$ in $A_{0}$ and $3 \%$ in $\gamma_{\mu}$.

As pointed out in [27], the vertical sea-level muon spectrum is not well known at energies greater than a few hundred $\mathrm{GeV}$; for energies below this range, the statistical and systematic errors of existing experiments are large $( \pm 10-15 \%)$. Hence our determination of the surface muon flux in the energy range $1<E_{p}<20 \mathrm{TeV}$ provides new information on the high-energy dependence of the sea-level muon spectrum.

In Fig. 6(a) the world data on the surface muon differential flux versus muon energy are presented. The solid line is the fit through our data. In Fig. 6(b) the same data are presented multiplied by $E_{\mu}^{3}$. Our fit agrees with the high-energy measurements at sea level. The same figure also shows the differential flux calculation of Ref. [1]. A maximum difference of $10 \%$ from our result is observed
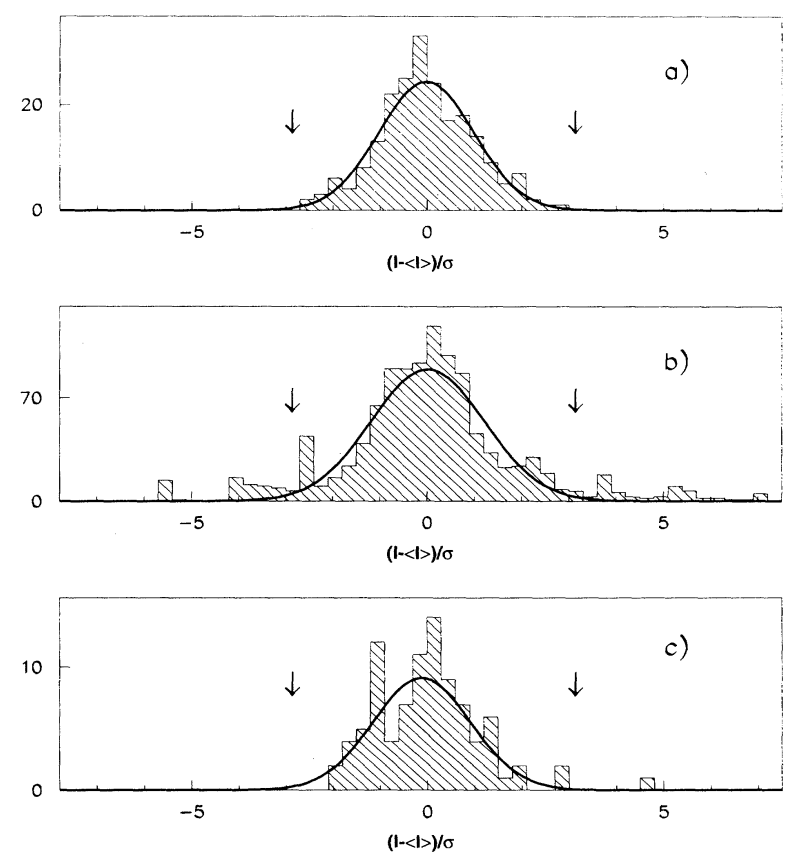

FIG. 7. Distributions of $[(I(h, \theta, \phi)-\langle I(h, \theta, \phi)\rangle) / \sigma]$ at fixed nominal depth $h$ measured in different angular bins. (a) $h=3200 \mathrm{hg} \mathrm{cm}^{-2}$, (b) $h=3800 \mathrm{hg} \mathrm{cm}^{-2}$, (c) $h=5600$ $\mathrm{hg} \mathrm{cm}^{-2}$. The solid lines represent Gaussian fits. Points outside \pm 3 standard deviations have been rejected. 
TABLE V. Gran Sasso rock thickness (m) as a function of zenith and azimuth (deg) for the mountain regions where the slant depth of the overburden is known with confidence.

\begin{tabular}{|c|c|c|c|c|c|c|c|c|c|c|c|c|}
\hline \multirow{2}{*}{$\frac{\text { Azimut }}{\text { (deg.) }}$} & \multicolumn{11}{|c|}{ Zenith angle (deg) } & \multirow[b]{2}{*}{60} \\
\hline & 5 & 10 & 15 & 20 & 25 & 30 & 35 & 40 & 45 & 50 & 55 & \\
\hline 0 & 1338 & 1302 & 1326 & 1292 & 1268 & 1292 & 1316 & 1324 & & & & \\
\hline 5 & 1339 & 1303 & 1302 & 1272 & 1244 & 1267 & 1303 & 1304 & & & & \\
\hline 10 & 1341 & 1305 & 1287 & 1254 & 1224 & 1246 & 1276 & 1295 & & & & \\
\hline 15 & 1343 & 1305 & 1278 & 1239 & 1216 & 1221 & 1238 & 1267 & & & & \\
\hline 20 & 1346 & 1307 & 1279 & 1237 & 1222 & 1240 & 1239 & 1234 & & & & \\
\hline 25 & 1349 & 1310 & 1280 & 1247 & 1248 & 1272 & 1263 & 1246 & & & & \\
\hline 30 & 1349 & 1313 & 1285 & 1271 & 1279 & 1309 & 1270 & 1277 & & & & \\
\hline 35 & 1350 & 1317 & 1291 & 1296 & 1301 & 1298 & 1303 & 1310 & & & & \\
\hline 40 & 1354 & 1323 & 1312 & 1320 & 1311 & 1311 & 1335 & 1343 & & & & \\
\hline 45 & 1361 & 1336 & 1335 & 1341 & 1326 & 1344 & 1352 & 1377 & & & & \\
\hline 50 & 1370 & 1350 & 1362 & 1374 & 1350 & 1372 & 1371 & 1378 & & & & \\
\hline 55 & 1376 & 1359 & 1386 & 1406 & 1375 & 1364 & 1380 & 1378 & & & & \\
\hline 60 & & & & 1414 & 1380 & 1352 & 1366 & 1370 & & & & \\
\hline 65 & & & & 1401 & 1374 & 1337 & 1350 & 1377 & & & & \\
\hline 70 & & & & 1390 & 1365 & 1347 & 1366 & 1383 & 1438 & 1487 & 1539 & 1597 \\
\hline 75 & & & & 1388 & 1371 & 1371 & 1384 & 1419 & 1471 & 1516 & 1562 & 1659 \\
\hline 80 & & & & 1398 & 1387 & 1388 & 1406 & 1465 & 1496 & 1533 & 1639 & 1774 \\
\hline 85 & & & & 1411 & 1405 & 1414 & 1448 & 1480 & 1501 & 1592 & 1726 & 1869 \\
\hline 90 & & & & 1420 & 1426 & 1446 & 1481 & 1495 & 1547 & 1674 & 1804 & 1908 \\
\hline 95 & & & & 1401 & 1400 & 1430 & 1472 & 1513 & 1585 & 1649 & 1754 & 1904 \\
\hline 100 & 1381 & 1367 & 1353 & 1380 & 1372 & 1395 & 1435 & 1482 & 1545 & 1598 & 1668 & 1805 \\
\hline 105 & 1377 & 1358 & 1328 & 1352 & 1361 & 1353 & 1401 & 1427 & 1486 & 1557 & 1615 & 1760 \\
\hline 110 & 1371 & 1350 & 1311 & 1330 & 1335 & 1345 & 1355 & 1398 & 1440 & 1495 & & \\
\hline 115 & 1367 & 1341 & 1302 & 1308 & 1310 & 1317 & 1320 & 1351 & 1392 & 1455 & & \\
\hline 120 & 1362 & 1330 & 1291 & 1280 & 1283 & 1286 & 1299 & 1317 & 1362 & 1431 & & \\
\hline 125 & 1356 & 1314 & 1284 & 1244 & 1255 & 1255 & 1269 & 1291 & 1338 & 1410 & & \\
\hline 130 & 1354 & 1304 & 1277 & 1232 & 1233 & 1234 & 1251 & 1268 & 1318 & 1397 & & \\
\hline 135 & 1350 & 1287 & 1268 & 1229 & 1211 & 1214 & 1225 & 1255 & 1306 & 1380 & & \\
\hline 140 & 1348 & 1287 & 1260 & 1223 & 1204 & 1199 & 1216 & 1246 & 1302 & 1375 & & \\
\hline 145 & 1346 & 1284 & 1250 & 1220 & 1193 & 1190 & 1207 & 1244 & 1296 & 1370 & & \\
\hline 150 & 1348 & 1283 & 1238 & 1215 & 1186 & 1182 & 1200 & 1241 & 1295 & 1371 & & \\
\hline 155 & 1347 & 1280 & 1235 & 1205 & 1179 & 1173 & 1200 & 1237 & 1297 & 1371 & & \\
\hline 160 & 1345 & 1277 & 1230 & 1203 & 1176 & 1168 & 1197 & 1236 & 1299 & 1378 & 1536 & 1764 \\
\hline 165 & 1346 & 1271 & 1229 & 1193 & 1172 & 1164 & 1196 & 1242 & 1297 & 1385 & 1562 & 1885 \\
\hline 170 & 1348 & 1269 & 1228 & 1185 & 1167 & 1166 & 1196 & 1250 & 1306 & 1401 & 1577 & 1974 \\
\hline 175 & 1349 & 1268 & 1227 & 1188 & 1166 & 1165 & 1196 & 1256 & 1320 & 1414 & 1599 & 2146 \\
\hline 180 & 1350 & 1268 & 1224 & 1192 & 1169 & 1166 & 1203 & 1260 & 1333 & 1429 & 1634 & 2142 \\
\hline 185 & 1348 & 1268 & 1222 & 1185 & 1173 & 1171 & 1208 & 1265 & 1347 & 1451 & 1670 & 2168 \\
\hline 190 & 1347 & 1266 & 1220 & 1183 & 1170 & 1178 & 1216 & 1271 & 1347 & 1470 & 1704 & 2085 \\
\hline 195 & 1342 & 1260 & 1220 & 1187 & 1172 & 1186 & 1222 & 1288 & 1363 & 1486 & 1716 & 2096 \\
\hline 200 & 1339 & 1264 & 1218 & 1186 & 1180 & 1193 & 1232 & 1298 & 1376 & 1504 & 1727 & 2023 \\
\hline 205 & 1345 & 1266 & 1216 & 1191 & 1188 & 1203 & 1241 & 1299 & 1384 & 1521 & 1738 & 2016 \\
\hline 210 & 1349 & 1272 & 1227 & 1198 & 1194 & 1218 & 1254 & 1307 & 1398 & 1559 & 1781 & 2243 \\
\hline 215 & 1352 & 1277 & 1239 & 1204 & 1207 & 1228 & 1262 & 1322 & 1406 & 1575 & 1866 & 2239 \\
\hline 220 & 1354 & 1282 & 1246 & 1210 & 1218 & 1240 & 1274 & 1334 & 1422 & 1597 & 1951 & 2227 \\
\hline 225 & 1356 & 1292 & 1254 & 1223 & 1230 & 1260 & 1301 & 1356 & 1456 & 1637 & 1989 & 2220 \\
\hline 230 & 1359 & 1308 & 1264 & 1251 & 1244 & 1280 & 1331 & 1397 & 1484 & 1665 & 2008 & 2225 \\
\hline 235 & 1363 & 1322 & 1278 & 1269 & 1263 & 1285 & 1340 & 1415 & 1497 & 1679 & 2051 & 2209 \\
\hline 240 & 1371 & 1337 & 1296 & 1292 & 1283 & 1300 & 1348 & 1424 & 1537 & 1741 & 2105 & 2286 \\
\hline 245 & 1379 & 1337 & 1317 & 1313 & 1300 & 1317 & 1360 & 1440 & 1583 & 1784 & 2195 & 2520 \\
\hline 250 & 1385 & 1341 & 1346 & 1332 & 1318 & 1343 & 1382 & 1462 & 1638 & 1843 & 2353 & 2630 \\
\hline 255 & 1392 & 1348 & 1366 & 1349 & 1342 & 1369 & 1422 & 1523 & 1766 & 2042 & 2454 & 2714 \\
\hline 260 & 1396 & 1364 & 1388 & 1368 & 1368 & 1396 & 1454 & 1567 & 1921 & 2178 & 2324 & 2520 \\
\hline 265 & & & & 1388 & 1396 & 1426 & 1476 & 1581 & 1968 & 2056 & 2195 & 2369 \\
\hline 270 & & & & 1409 & 1433 & 1457 & 1505 & 1587 & 1889 & 2021 & 2120 & 2303 \\
\hline 275 & & & & 1439 & 1470 & 1499 & 1538 & 1616 & 1890 & 2012 & 2133 & 2346 \\
\hline 280 & & & & 1465 & 1499 & 1526 & 1560 & 1639 & 1897 & 1998 & 2177 & 2394 \\
\hline 285 & & & & 1499 & 1523 & 1560 & 1579 & 1656 & 1888 & 1996 & 2199 & 2426 \\
\hline
\end{tabular}


TABLE V. (Continued).

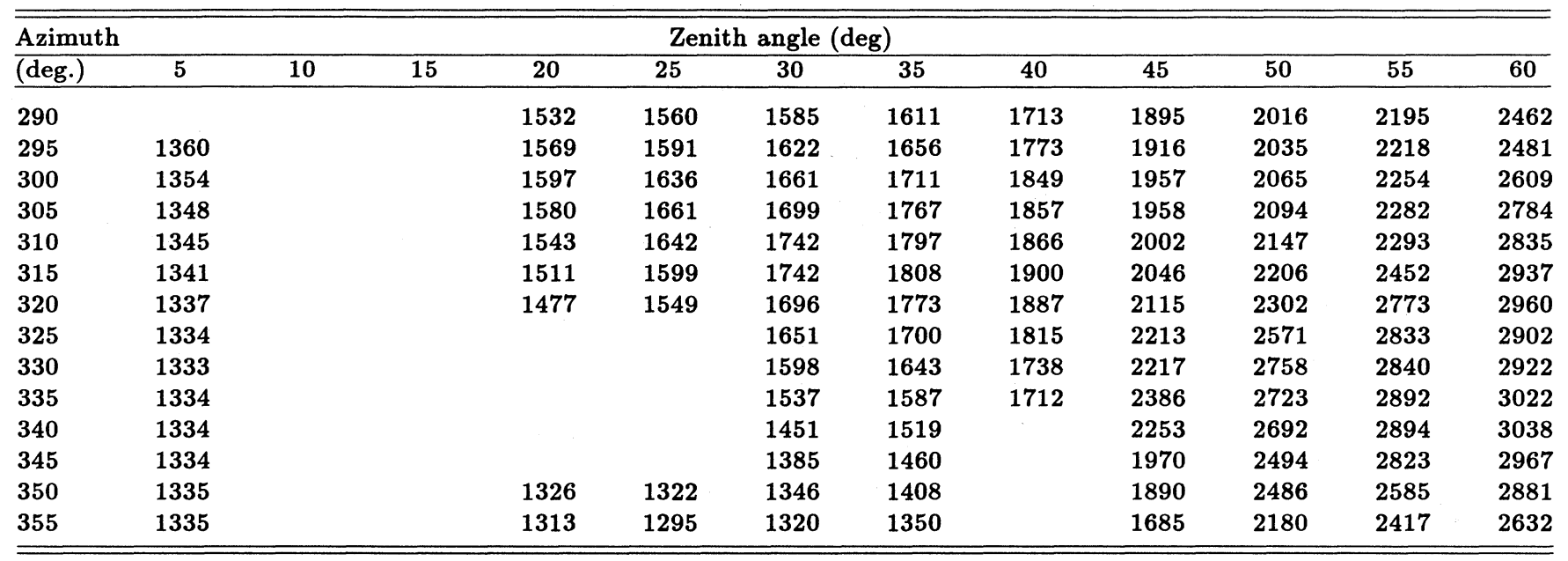

at $1 \mathrm{TeV}$. Our data provide new information on the flux of muons above $1 \mathrm{TeV}$.

\section{CONCLUSIONS}

We measured the underground muon intensity as a function of the slant depth, in the range 3200-7000 hg $\mathrm{cm}^{-2}$. The average parameters of the rock were estimated using the material extracted during the tunnel excavation and the mountain surveys (see the Appendix). The high statistics of this data sample allowed identification of regions where the mountain map is not well known. Our vertical muon intensities agree with the SOUDAN [18] and BAKSAN [6(g)] data and the world compilation of Ref. [17]; the Frejus [16] and the NUSEX [8] data are lower.

Using three different nuclear interaction models the primary "all-nucleon" spectrum has been evaluated in the energy range $2<E_{p}<200 \mathrm{TeV} /$ nucleon; it is compatible with the average of the available direct measurements in this energy range. The spectral index is almost model independent while the spread in the absolute normalization is about $25 \%$ larger than the statistical uncertainty.

From our data we determined the surface muon flux. For muon energies larger than $1 \mathrm{TeV}$, our data agree with the analytical estimate of Ref. [1].

\section{ACKNOWLEDGMENTS}

We gratefully acknowledge the support of the director and of the staff of the Laboratori Nazionali del Gran Sasso and the invaluable assistance of the technical staff of the Institutions participating in the experiment. We thank the Istituto Nazionale di Fisica Nucleare (INFN), the U.S. Department of Energy, and the U.S. National Science Foundation for their generous support of the MACRO experiment. We thank INFN for providing financial support (FAI) for non Italian citizens.

\section{APPENDIX: CHARACTERISTICS OF THE GRAN SASSO ROCK}

The rock surrounding the Gran Sasso underground laboratory has an irregular structure; its composition is essentially calcareous, mixed with other materials, such as aluminum, silicon, magnesium compounds, and organic remains. Detailed analyses were made of the material obtained during the tunnel excavation. It was thus possible to make a composition and density model of the Gran Sasso rock [14]. The chemical composition is given in Table III.

The average values of the elemental composition parameters were calculated in the angular range $0^{\circ}-60^{\circ}$; they are very close to the standard rock values (see Table IV). The correction to go from Gran Sasso to standard rock was applied following Ref. [13].

To evaluate the vertical muon intensity, the muon data were divided in angular bins $\Delta \theta=1^{\circ}, \Delta \phi=2^{\circ}$. For each bin a nominal rock thickness from the digitization of the mountain topographic map supplied by the Italian Military Geographical Institute (IGM) and a vertical muon intensity were evaluated. The distributions of the intensities for each angular bin exhibited, at fixed nominal depth, a Gaussian behavior. Some points are outside three standard deviations from the average as can be seen in Fig. 7 where the distribution in the quantity $[I((h, \theta, \phi)-\langle I(h, \theta, \phi)\rangle) / \sigma]$ is shown for three nominal depths. Figure 7 is an example of the study of the point to point uncertainties performed. In Fig. 7(b) the angular regions which yield muon intensities deviating more than $\pm 3 \sigma$ from the average in the same slant depth are clearly visible. These regions have been identified and rejected. The data sample was reduced to $2.62 \times 10^{6}$ muons. In Table V the Gran Sasso rock thicknesses surviving the angular cuts previously described, are given as a function of the zenith and the azimuth angles. The azimuth is measured relative to geographic north. The empty bins represent the angular rejected regions according to the above criteria. 
[1] T. Gaisser, Cosmic Rays and Particle Physics (Cambridge University Press, Cambridge, England, 1990).

[2] S.P. Ahlen et al., Phys. Rev. D 46, 895 (1992).

[3] S.P. Ahlen et al., Phys. Rev. D 46, 4836 (1992).

[4] M. Aglietta et al., Phys. Lett. B 337, 376 (1994).

[5] K. Asakimori et al., in Cosmic Ray Conference, Proceedings of the 23rd International Conference, Calgary, Canada, 1993, edited by R. B. Hicks et al. (World Scientific, Singapore, 1994), Vol. 2, pp. 21 and 25; S. Swordy, ibid., p. 243.

[6] (a) C. Castagnoli et al., Nuovo Cimento A 82, 78 (1984); (b) A. Castellina et al., ibid. C 8, 93 (1985); (c) L. Bergamasco et al., ibid. 6, 596 (1983); (d) P.H. Barrett et al., Rev. Mod. Phys. 24, 133 (1952); (e) L.M. Bollinger, Phys. Rev. 79, 207 (1950); (f) R.I. Enikeev et al., Sov. J. Nucl. Phys. 47, 665 (1988); (g) Y.M. Andreyev, V.I. Gurentsov, and I.M. Kogai, in Proceedings of the 20th International Cosmic Ray Conference, Moscow, USSR, 1987, edited by V. Kozyarivsky et al. (Nauka, Moscow, 1987), Vol. HE 4.1-19; (h) V.N. Bakatanov et al., Sov. J. Nucl. Phys. 55, 1169 (1992).

[7] S.P. Ahlen et al., Phys. Lett. B 249, 149 (1990).

[8] M. Aglietta et al., in Astrophysics and Particle Physics, Proceedings of the Topical Seminar, San Miniato, Italy, 1989, edited by G. Castellini et al. [Nucl. Phys. B (Proc. Suppl.) 14, 193 (1990)].

[9] H. Adarkar et al., in Proceedings of the 21st International Cosmic Ray Conference, Adelaide, Australia, 1989, edited by R. J. Protheroe (Graphic Services, Northfield, South Australia, 1990), Vol. 9, p. 310.

[10] S.P. Ahlen et al., Nucl. Instrum. Methods A 234, 337 (1993).

[11] S.P. Ahlen et al., Astrophys. J. 412, 301 (1993).

[12] R. Brun et al., "CERN GEANT 3 User's Guide," Report No. DD/EE/84-1, 1992 (unpublished).

[13] Yu. D. Kotov and V. M. Logunov, in Proceedings of the 11th International Cosmic Ray Conference, Budapest, Hungary, 1969, edited by A. Somogyi [Acta Phys. Acad.
Sci. Hung. Suppl. 29 (1970)].

[14] P.G. Catalano, "Caratteristiche geolitologiche e strutturali dell'ammasso roccioso sovrastante il laboratorio I.N.F.N.," ANAS report, 1986 (unpublished); P.G. Catalano et al., Mem. Soc. Geol. It. 35, 647 (1986).

[15] H. Bilokon et al., "Muon survival probabilities in the Gran Sasso Rock," Report No. LNGS-94/92, 1994 (unpublished).

[16] Ch. Berger et al., Phys. Rev. D 40, 2163 (1989).

[17] M. Crouch, in Proceedings of the 20th International Cosmic Ray Conference [6], Vol. 6, p. 165.

[18] K. Ruddick (private communication); Soudan Collaboration, Int. Report No. PDK-435, 1990 (unpublished); S.M. Kasahara, Ph.D. thesis, University of Minnesota, 1995.

[19] C. Forti et al., Phys. Rev. D 25, 3668 (1990).

[20] R.S. Fletcher, T.K. Gaisser, P. Lipari, and T. Stanev, Phys. Rev. D 50, 5710 (1994).

[21] L. Bergamasco et al., Nuovo Cimento C 6, 569 (1983).

[22] G. Parente, A. Shoup, and G.B. Yodh, Astropart. Phys. 3, 17 (1995).

[23] MACRO Collaboration, M. Ambrosio et al., in Cosmic Ray Conference [5], Vol. 2, p. 97.

[24] R.P. Kokoulin and A.A. Petrukin, in Cosmic Ray Conference, Proceedings of the 22nd International Conference, Dublin, 1991 (World Scientific, Singapore, 1991), Vol. 4, p. 536.

[25] P. Lipari and T. Stanev, Phys. Rev. D 44, 3543 (1991).

[26] W. Lohmann, R. Kopp, and R. Voss, "Energy losses of muons in the energy range $1-10000 \mathrm{GeV}$," Report No. CERN 85-03, 1985 (unpublished).

[27] D.H. Perkins, Nucl. Phys. B399, 3 (1993).

[28] O.C. Allkofer, K. Carstensen, and W.D. Dau, Phys. Lett. 36B, 425 (1971).

[29] C.A. Ayre et al., J. Phys. G 1, 584 (1975).

[30] P.J. Green et al., Phys. Rev. D 20, 1598 (1979).

[31] B.C. Nandi and M.S. Sinha, J. Phys. A 5, 1384 (1972).

[32] B.C. Rastin, J. Phys. G 10, 1609 (1984). 